\title{
Editorial
}

\section{Advances in Low-Profile Antennas in Wireless Communications 2015}

\author{
Guo Qing Luo, ${ }^{1}$ Xiao Ping Chen, ${ }^{2}$ Zhang Cheng Hao, ${ }^{3}$ Bing Liu, ${ }^{4}$ and Yu Jian Cheng ${ }^{5}$ \\ ${ }^{1}$ School of Electronics and Information, Hangzhou Dianzi University, Hangzhou 310018, China \\ ${ }^{2}$ Poly-Grames Research Centre, Department of Electrical Engineering, University of Montreal, Montreal, QC, Canada H3T 1J4 \\ ${ }^{3}$ School of Information Science and Engineering, Southeast University, Nanjing 210096, China \\ ${ }^{4}$ College of Electronic and Information Engineering, Nanjing University of Aeronautics and Astronautics, Nanjing 210016, China \\ ${ }^{5}$ School of Electronic Engineering, University of Electronic Science and Technology of China, Chengdu 611731, China
}

Correspondence should be addressed to Guo Qing Luo; gqluo@emfield.org

Received 7 September 2015; Accepted 7 September 2015

Copyright (c) 2015 Guo Qing Luo et al. This is an open access article distributed under the Creative Commons Attribution License, which permits unrestricted use, distribution, and reproduction in any medium, provided the original work is properly cited.

Although low-profile antennas designs have been widely studied in the past years, there are still many challenging issues in low-profile antennas in wireless communications. In this special issue, the accepted papers include the designs of multiple frequency antenna, conformal substrate integrated waveguide (SIW) antenna, membrane antenna, reconfigurable antenna, on-chip antenna, and handset antenna. Their operating frequency ranges cover from several hundred $\mathrm{MHz}$ to several hundred THz.

T. Fukusako (Kumamoto University of Japan) and colleagues proposed a low-profile and compact printed antenna having an omnidirectional radiation pattern with horizontal polarization to the ground. The proposed antenna consists of an inner small fed ring, an outer coupled split ring, and a ground plane. The proposed antenna covers the $920 \mathrm{MHz}$ RFID band, and its gain is $1.45 \mathrm{dBi}$ in the parallel direction to the ground plane.

H. Liu et al. (Xidian University of China) proposed a novel octaband LTE mobile phone antenna. The miniaturized octaband antenna is implemented by a simple prototype of three parts which include a folded monopole as feeding element, main radiator element, and parasitic radiator element. The main and parasitic radiator elements are excited by the folded monopole feeding element coupling and shorting to the handset ground plane. The antenna has the total efficiency up to $30 \%$ in low bands and up to $75 \%$ in high bands, respectively.
A. S. Mekki et al. (Universiti Putra Malaysia of Malaysia) proposed a gain enhanced and size reduced microstrip patch antenna. The proposed unidirectional and low-profile microstrip patch antenna of $0.069 \lambda_{0}$ operates at $2.45 \mathrm{GHz}$ and has an impedance bandwidth of $2 \%$, a gain of $5.2 \mathrm{dBi}$, and a front to back ratio of $9.5 \mathrm{~dB}$.

Y. Li (Harbin Engineering University of China) and W. Yu proposed a miniaturized triple band monopole antenna for microwave access (WiMAX) and wireless local area network (WLAN) communication applications. Its three resonance frequencies are realized by using a toothbrush-shaped patch (TSP), a meander line (ML), and an inverted U-shaped patch (IUSP). The center frequencies of the triple bands can be controlled by adjusting the dimensions of the TSP, ML, and IUSP, respectively.

S. S. Punjala (Tata Consultancy Services of India) and colleagues proposed a novel broadband reconfigurable antenna covering different frequency bands for wireless powering application in a concrete block. The reconfigurable antenna contains a circular patch, three concentric circular rings around the circular patch, and RF MEMS switches connected to the neighbored circular rings.

W. N. Huang et al. (University of Electronic Science and Technology of China) proposed a conical conformal leaky-wave antenna based on SIW technology. This antenna conforms to a conical shape surface with the angle of $40^{\circ}$. It has a narrow beam that scans from $80^{\circ}$ to $97^{\circ}$ with varying frequency from $34 \mathrm{GHz}$ to $37 \mathrm{GHz}$. 
H. Vettikalladi from King Saud University of Saudi Arabia proposed a single slot coupled SIW fed membrane antenna loaded with a superstrate layer for $94 \mathrm{GHz}$ communication system. The membrane antenna consists of 6 layers. The microstrip patch antenna (MPA) placed on the top substrate layer is excited by means of a longitudinal rectangular slot placed over the SIW structure in the bottom substrate. The maximum gain achieved is $14.4 \mathrm{dBi}$ with an efficiency of $77.6 \%$ at $94 \mathrm{GHz}$.

M. Himdi (University of Rennes of France) and colleagues proposed an equilateral triangular dielectric resonator nanoantenna at optical frequencies for solar energy harvesting application. The proposed antenna is composed of an "Ag- $\mathrm{SiO}_{2}-\mathrm{Ag}$ " structure with a nanosilver "Ag" transmission line that excites a triangular dielectric made of "Si" material. It has a wide impedance bandwidth of $2.58 \%$ with a high directive radiation pattern of $8.6 \mathrm{dBi}$ at $193.5 \mathrm{THz}$ with an end-fire radiation pattern.

We hope that, through this special issue, the readers will find not only new designs about different low-profile antennas but also their valuable applications.

\title{
Acknowledgment
}

The editors thank all the contributors and the anonymous reviewers for their contributions to this special issue.

\author{
Guo Qing Luo \\ Xiao Ping Chen \\ Zhang Cheng Hao \\ Bing Liu \\ Yu Jian Cheng
}



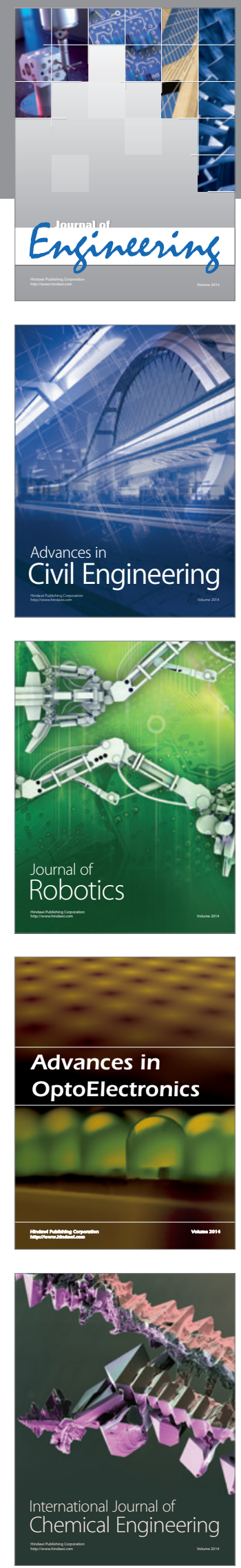

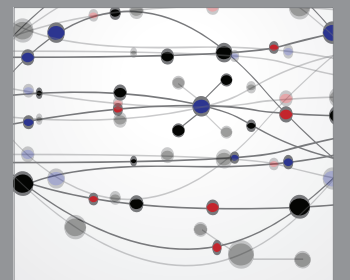

The Scientific World Journal
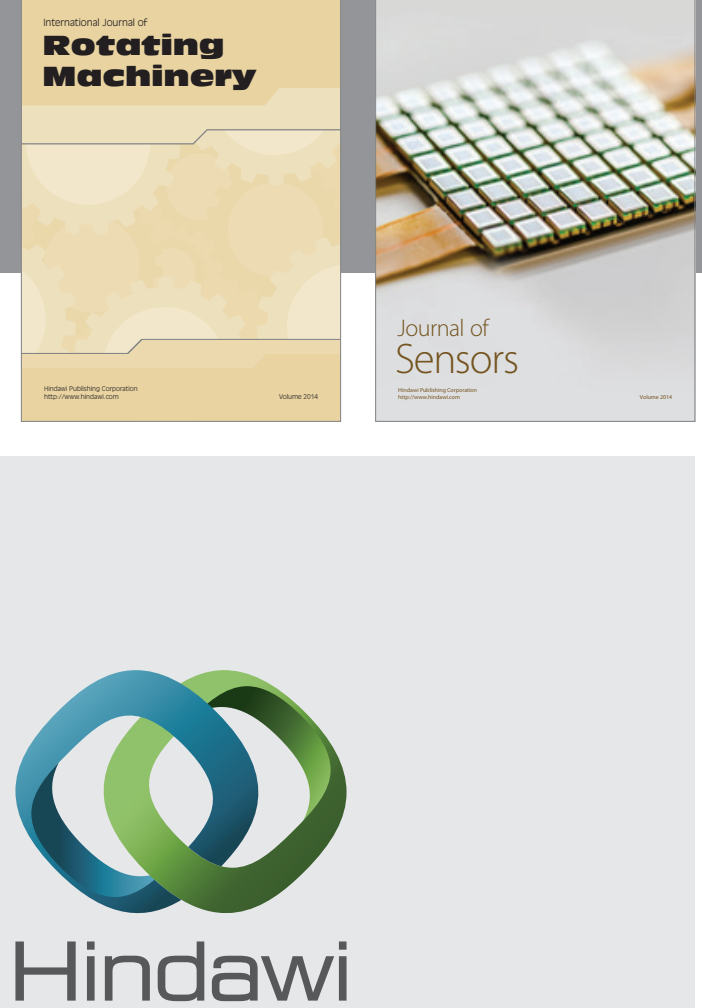

Submit your manuscripts at http://www.hindawi.com
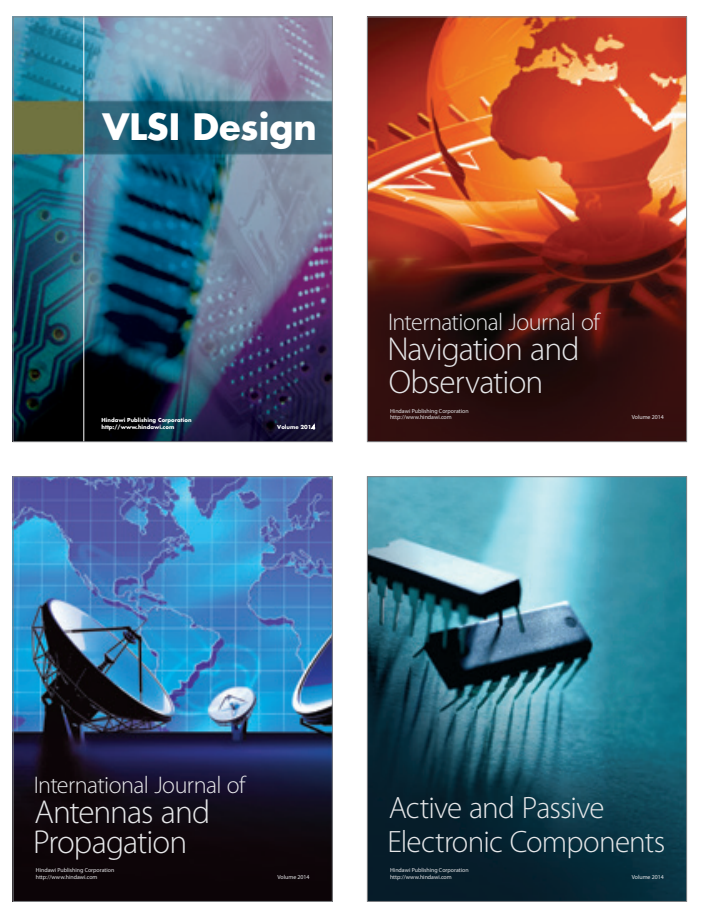
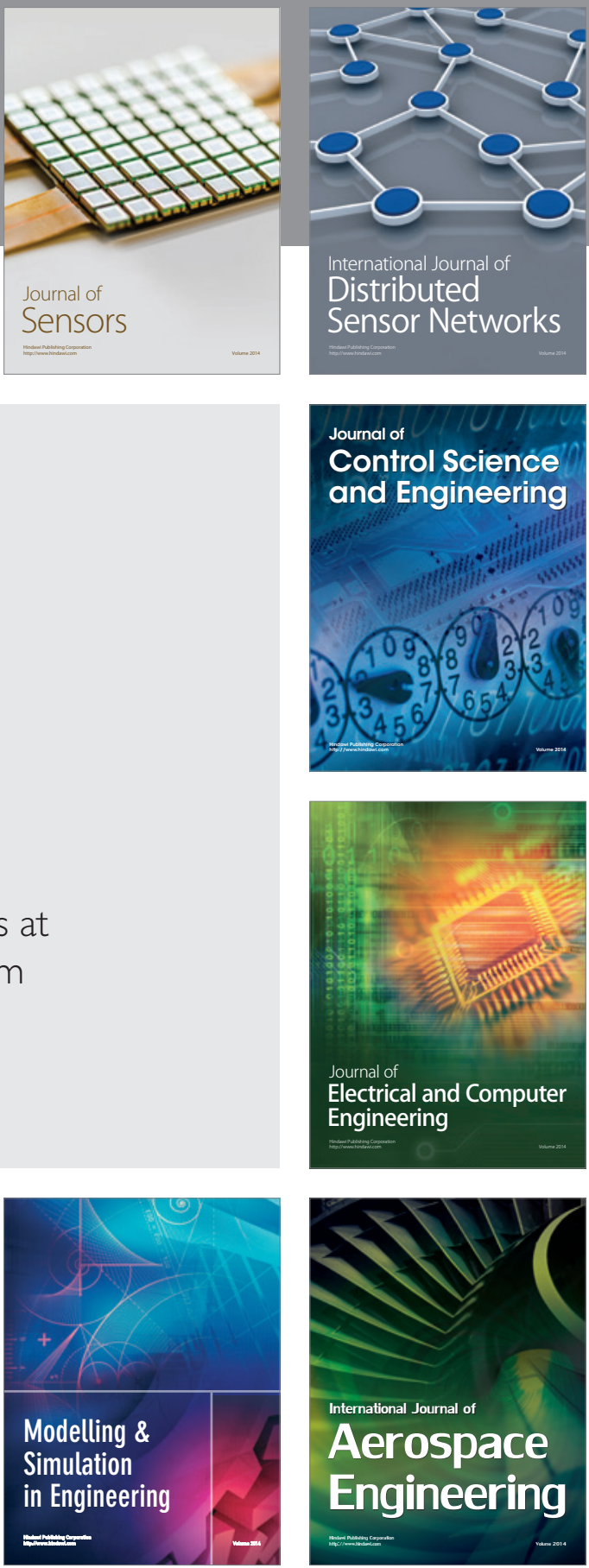

Journal of

Control Science

and Engineering
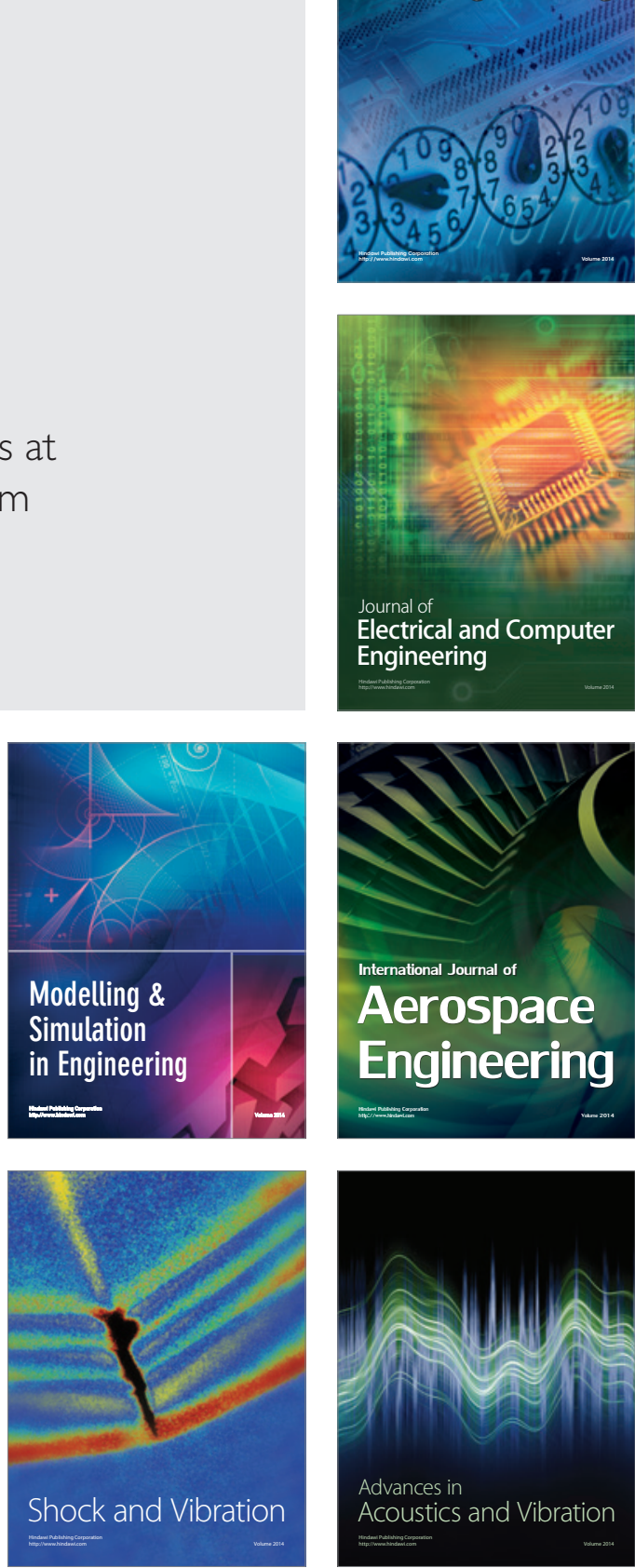